\title{
Intra-Articular Autologous Platelet-Rich Plasma Hip Injection May Result in Osteogenesis Depicted as an Increase in Femoral Neck Bone Density*
}

\author{
Marwa A. Ahmed, Andre Panagos \\ Spine and Sports Medicine of New York, New York, USA \\ Department of Rehabilitation Medicine, NYU Langone Medical Center, New York, USA \\ Email: ma@ssmny.com
}

Received 16 March 2014; received 12 April 2014; accepted 18 April 2014

Copyright (C) 2014 by authors and Scientific Research Publishing Inc.

This work is licensed under the Creative Commons Attribution International License (CC BY). http://creativecommons.org/licenses/by/4.0/

(c) $\underset{\mathrm{EY}}{\mathrm{B}}$ Open Access

\begin{abstract}
Osteoporosis has become one of the most prevalent bone diseases in developed countries. As the world's population ages, this complex skeletal disorder is predicted to become an even more widespread and serious health condition, posing greater societal economic burden. Current therapeutic agents are limited by clinically significant side effects. In this report, we present a case that suggests intra-articular platelet-rich plasma (PRP) injectate may improve bone mineral density (BMD). This observation raises a question with exciting implications-can PRP serve as a viable and relatively safe, targeted treatment option to improve bone density?
\end{abstract}

\section{Keywords}

Platelet-Rich Plasma, Osteogenesis, Osteoporosis, Bone Density

\section{Introduction}

Platelet-rich plasma (PRP) is a form of treatment that has shown an exciting promise with regard to optimal and long-lasting hard and soft tissue healing in various fields of medicine, including sports medicine, orthopedics, ENT, ophthalmology, dentistry and maxillofacial surgery. PRP has long had roots in veterinary medicine and its

*This material has NOT been presented at any assembly.

No funding sources.

Informed patient consent was obtained.

How to cite this paper: Ahmed, M.A. and Panagos, A. (2014) Intra-Articular Autologous Platelet-Rich Plasma Hip Injection May Result in Osteogenesis Depicted as an Increase in Femoral Neck Bone Density. Open Journal of Regenerative Medicine, 3, 39-42. http://dx.doi.org/10.4236/ojrm.2014.32005 
application for dental procedures predates its use in the field of sports medicine by nearly a decade [1].

PRP is an autologous concentration of platelets in concentrated plasma. Platelets are a rich source of cytokines, interleukin 8 and growth factors including platelet-derived growth factor (PDGF), transforming growth factor (TGF), vascular endothelial growth factor (VEGF) and insulin-like growth factor (IGF). By concentrating growth factors, PRP promotes the healing potential of the body and accelerates the regeneration process [2].

The application of PRP for the prevention or treatment of osteoporosis has been a looming question with exciting implications. Osteoporosis is a complex bone disease that has become one of the most prevalent skeletal disorders in developed countries. It is characterized by low bone mass and microarchitectural deterioration of the bones, reflecting a disruption in the bone remodeling process. This results in bone fragility and an increased risk of fracture [3].

The current available strategies to treating osteoporosis target bone resorption inhibition by inhibiting osteoblast activity. The four major anti-resorptive agents used in clinics are estrogen, selective estrogen receptor modulators, bisphosphonates and calcitonin. While these agents have proven to be effective, their long-term use is limited by serious side effects [4]. Furthermore, these agents cannot promote bone formation. As our population ages, it is likely that osteoporosis will become an even more prevalent and serious health condition, posing a greater societal economic burden [3]. The research and development of other more effective therapeutic agents with an improved safety profile are thus imperative.

We report a case of an osteopenic female who was incidentally found to have a $12.2 \%$ increase in femoral BMD for several months following an ipsilateral autologous platelet-rich plasma intra-articular hip injection.

\section{Case Presentation}

The patient is a 65 year-old female with a long-standing history of left groin pain related to hip joint osteoarthritis. Plain films of the left hip joint revealed minimal degenerative change (Figure 1), while moderate to severe degenerative changes with labral degeneration were noted on MRI. She received several intra-articular corticosteroid hip injections over a two-year span with significant improvement in her symptoms, though short-lived and lasting only a few weeks at a time. Clinical management was subsequently escalated and she was treated with an autologous platelet-rich plasma intra-articular left hip injection (7/29/2013). At one month post-procedure, an 85\% improvement was noted in her symptoms and this same improvement continued to most recently, 8 months post-procedure.

Comparative DEXA bone density measurements of the left femoral neck showed a T-score range from -2.7 to -2.2 over the ages of 51 - 63 (Table 1). DEXA T-score at the age of 65 and 3 months post-PRP of the left hip showed a $12.2 \%$ increase as compared to DEXA results 2 years prior $(-2.49 / 2011 ;-1.811 / 2013)$. Of note, the patient was not taking any medications targeted to the prevention or treatment of osteopenia during this time with the exception of intermittent Vitamin D supplementation.

\section{Discussion}

While PRP's osteogenic properties are not novel, we believe that this report is the first to document a translation of this property as an increase in femoral neck BMD following an ipsilateral intra-articular PRP injection. A

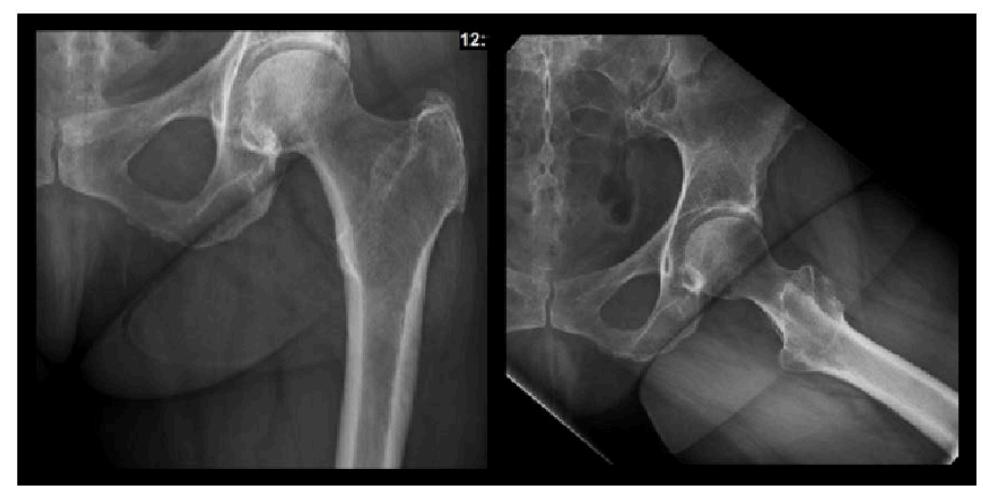

Figure 1. Left Hip X-Ray (AP and Frog Leg Views). AP and frog-leg plain films of the left hip joint revealing minimal degenerative change. 
Table 1. DEXA results summary.

\begin{tabular}{|c|c|c|c|c|c|}
\hline Scan Date & Age & $\operatorname{BMD}\left(\mathrm{g} / \mathrm{cm}^{2}\right)$ & T-Score & BMD Change vs. Baseline & BMD Change vs. Previous \\
\hline $11 / 01 / 2013$ & 65 & 0.654 & -1.8 & $8.7 \%$ & $12.2 \%$ \\
\hline 09/23/2011 & 63 & 0.583 & -2.4 & $-3.2 \%$ & $-0.7 \%$ \\
\hline $10 / 09 / 2008$ & 60 & 0.587 & -2.4 & $-2.5 \%$ & $6.8 \%$ \\
\hline 06/20/2006 & 57 & 0.549 & -2.7 & $-8.8 \%$ & $-8.4 \%$ \\
\hline 08/26/2003 & 54 & 0.599 & -2.3 & $-0.4 \%$ & $-0.4 \%$ \\
\hline 09/21/1999 & 51 & 0.602 & -2.2 & N/A & N/A \\
\hline
\end{tabular}

literature review using PubMed displayed various articles related to in-vivo, human studies of post-operative oral and maxillofacial bone healing [2] [5]. Studies that looked into PRP's potential role in the specific prevention and treatment of osteoporosis were animal rather than human based [3].

The DEXA results of the patient described above show a $6.8 \%$ increase in left femoral neck BMD from 2006-2008 (T scores: -2.7 to -2.4 ) and a larger 12.2\% increase from 2011-2013 (T scores: -2.4 to -1.8 ). We are unable to explain the increase noted across 2006-2008, however we did verify that other than intermittent supplementation with Vitamin D over the years, the patient has not been taking any targeted treatments to improve BMD. She has been on hormone replacement therapy (Estradiol transdermal patches) over the past 4-5 years to treat symptoms of menopause. While hormone replacement is known to slow bone turnover and increase BMD, the 6.8\% increase in her bone density across 2006-2008 does not coincide temporally with her usage. It is also unlikely that this therapy could explain the 12.2\% increase in BMD across 2011-2013 since her usage began around 2008 and one would expect a gradual increase in BMD rather than a $-0.7 \%$ decrease from 2008-2011 followed by the 12.2\% increase over 2011-2013. Lastly, while vitamin D has been shown to decrease the incidence of fractures in the osteoporotic population, studies have not shown it to have an effect on BMD [6]. We therefore suggest in this report, that the intra-articular PRP hip injection may account for the larger $12.2 \%$ increase in the ipsilateral femoral neck BMD. Should this be a true causal relationship, it may have a significant clinical application.

Fragility fractures are most common at the wrist, spinal vertebrae and hip. The incidence of hip and vertebral fractures increases exponentially with advancing age, while that of wrist fractures levels off after the age of 60 years [7]. The question raised is whether PRP could serve as a viable and relatively safe targeted treatment option to improve local BMD. To our knowledge, a literature review did not yield any information on other available local methods with this property. A limitation of the results presented in this report to consider is that we are unable to say whether this increase is indeed local to the injection region versus a more global increase in BMD. However, one would rationalize that the injection's effect would likely be more of a local one.

Bone remodeling is a complex process involving the close interactions between different progenitor cell lineages, mainly osteo- and adipo-progenitor cells. Several lines of evidence support this relationship between osteoblastogenesis and adipogenesis. Clinically, it has been demonstrated that the increased adipogenesis and fat content in the bone marrow is inversely correlated with decreased BMD in the elderly and osteoporotic population [3]. These findings indicate that osteoporosis could be the result of age-related infiltration of marrow fat, which suppresses osteoblast differentiation, function and survival [8]. Various signaling pathways with both local and systemic effects control the balance between osteoblast and adipocyte differentiation of mesenchymal stem cells [9].

A group of researchers in China, Lo et al., demonstrated that intraosseous transplantation of PRP-treated osteoblast-like cells into ovariectomized senescence-accelerated mice (OVX-SAMP8) prevented the development of osteoporosis [10]. PRP's ability to promote osteogenesis specifically involves but is not limited to PDGF and TGF-B1, which have been shown to promote the proliferation and differentiation of osteoblasts. TGF-B1 additionally inhibits the differentiation of adipocytes while IGF plays an important role in bone matrix synthesis and fat elimination. A later study by the same group confirmed and demonstrated that PRP treatment in this animal model mainly exerted its action via promoting bone regeneration with simultaneous suppression of adipogenesis within the marrow. "Collectively, PRP represents an ideal molecular modulator for osteogenesis” [3].

\section{Conclusion}

The use and clinical validation of PRP are still in its early stages. Further research is necessary in establishing a 
clear relationship between PRP and the prevention and/or treatment of osteoporosis in humans. With the progressive lengthening of the human life-span and advancing population age, osteoporotic fractures are predicted to become an even greater cause of morbidity, disability and in the case of hip fractures, even premature death. Furthermore, treatment of these fractures is currently already a considerable economic burden, costing billions of dollars per year [7]. PRP may be able to offer a safer but also potentially more localized treatment alternative to the available medications today, which have notoriously been known to increase the risk of developing atrial fibrillation and fractures related to bone abnormalities.

\section{References}

[1] Arora, N.S., et al. (2009) Platelet-Rich Plasma: A Literature Review. Implant Dentistry, 18, 303-310. http://dx.doi.org/10.1097/ID.0b013e31819e8ec6

[2] Marx, R.E. (2004) Platelet-Rich Plasma: Evidence to Support Its Use. Journal of Oral and Maxillofacial Surgery, 62:489-496. http://dx.doi.org/10.1016/j.joms.2003.12.003

[3] Liu, H., et al. (2011) The Balance between Adipogenesis and Osteogenesis in Bone Regeneration by Platelet-Rich Plasma for Age-Related Osteoporosis. Biomaterials, 32, 6773-6780. http://dx.doi.org/10.1016/j.biomaterials.2011.05.080

[4] Mitchner, N.A. and Harris, S.T. (2009) Current and Emerging Therapies for Osteoporosis. The Journal of Family Practice, 58, S45-S49.

[5] Marx, R.E., Carlson, E.R., Eichstaedt, R.M., et al. (1998) Platelet-Rich Plasma: Growth Factor Enhancement for Bone Grafts. Oral Surgery, Oral Medicine, Oral Pathology, Oral Radiology and Endodontology, 85, 638-646. http://dx.doi.org/10.1016/S1079-2104(98)90029-4

[6] Prentice, A. (2004) Diet, Nutrition and the Prevention of Osteoporosis. Public Health Nutrition, 7, 227-243.

[7] Ian R.R., et al. (2013) Effects of Vitamin D Supplements on Bone Mineral Density: A Systematic Review and Meta-Analysis. The Lancet, 383, 146-155.

[8] Duque, G. (2008) Bone and Fat Connection in Aging Bone. Current Opinion in Rheumatology, 20, 429-434. http://dx.doi.org/10.1097/BOR.0b013e3283025e9c

[9] Arvidson, K., et al. (2011) Bone Regeneration and Stem Cells. Journal of Cellular and Molecular Medicine, 15, 718-746. http://dx.doi.org/10.1111/j.1582-4934.2010.01224.x

[10] Lo, W.C., et al. (2009) Transplantation of Embryonic Fibroblasts Treated with Platelet-Rich Plasma Induces Osteogenesis in SAMP8 Mice Monitored by Molecular Imaging. Journal of Nuclear Medicine, 50, 765-773. http://dx.doi.org/10.2967/jnumed.108.057372

\section{Abbreviations}

PRP_Platelet-rich plasma

BMD_Bone mineral density

PDGF-Platelet-derived growth factor

TGF-Transforming growth factor

VEGF - Vascular endothelial growth factor

IGF-Insulin-like growth factor 
Scientific Research Publishing (SCIRP) is one of the largest Open Access journal publishers. It is currently publishing more than 200 open access, online, peer-reviewed journals covering a wide range of academic disciplines. SCIRP serves the worldwide academic communities and contributes to the progress and application of science with its publication.

Other selected journals from SCIRP are listed as below. Submit your manuscript to us via either submit@scirp.org or Online Submission Portal.
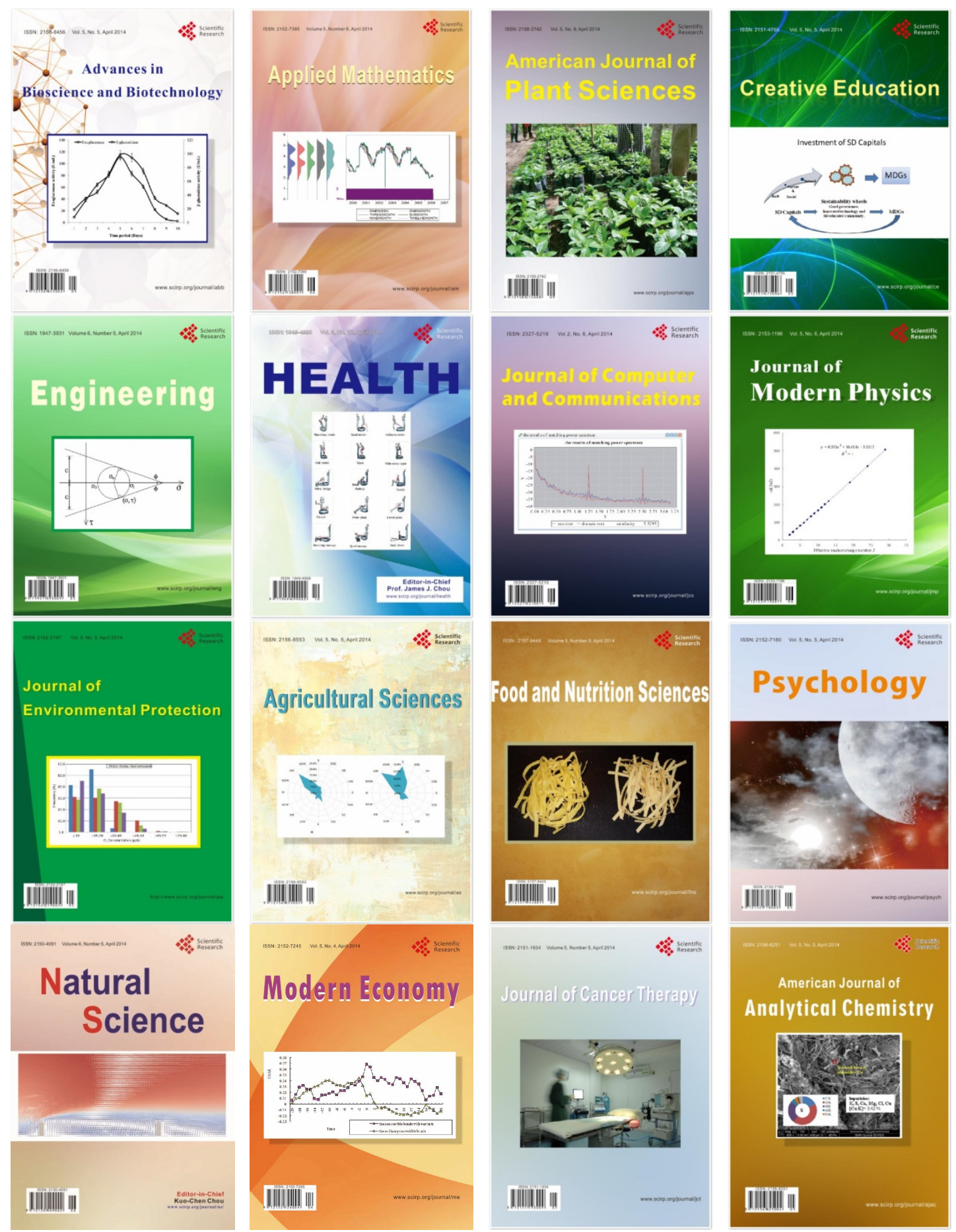